\title{
Health Conditions Related to Air Travel and Destination Country
}

\author{
Ülle Parm ${ }^{1 *}$, Grete Must ${ }^{1,2}$, Kätlin Kindsiko ${ }^{1,2}$ and Anna-Liisa Tamm ${ }^{1}$
}

${ }^{1}$ Physiotherapy and Environmental Health Department, University of Tartu, Estonia

${ }^{2}$ Department of Ergonomics, Institute of Technology, University of Life Sciences, Tartu, Estonia

*Corresponding author: Ülle Parm, Physiotherapy and Environmental Health Department, Tartu Health Care College, University of Tartu, Estonia, Nooruse 5, 50411 Tartu, Estonia, Tel: +37253487334; E-mail: ylleparm@nooruse.ee

Received date: April 26, 2018; Accepted date: April 01, 2018; Published date: May 07, 2018

Copyright: @ 2018 Parm Ü, et al. This is an open-access article distributed under the terms of the Creative Commons Attribution License, which permits unrestricted use, distribution, and reproduction in any medium, provided the original author and source are credited.

\begin{abstract}
Objective: Because of the increasing use of air travel and the increased mobility of people, different health conditions may arise during the trip in the flight cabin or in the destination country. We aimed to determine the health conditions/problems associated with air travel and stays in a foreign country.

Methods: The research included an electronic questionnaire completed by 1014 Estonian residents who travelled by plane during the last five years on holiday and/or on a cultural trip.

Results: Health disorders occurred mostly during long trips of 1-4 hours, and the main health problems during plane travel were earaches, headaches, and flatulence. Fear of flying occurred in nearly in a third of the subjects on both short- and long-distance flights. It manifested in episodes of tachycardia and pre-travel insomnia. To cope with these situations, female passengers preferred to listen to music, and male passengers preferred to consume alcohol. In total, 59 different countries were visited, and the most frequent destinations were southern countries: Turkey, Spain, and Italy. Diarrhea, sunburn, severe headaches, fevers and flu-like symptoms were the main health problems in the destination countries. These problems were mostly caused by bad hygiene, consumption of local food, not applying sunblock creams and the change of the environment. The major share of information about the potential dangers of a trip was received from the Internet or from friends.
\end{abstract}

Conclusions: People who decide to travel by plane are usually frequent flyers, and the preferred destinations were mostly warmer climates and not very distant but also not neighbor countries. Health problems occurred most frequently during flights of medium length (1-4 hours). The frequent health disorders at destinations were characteristic for northern tourists visiting southern countries.

Keywords: Travelling; Plane; Fear of flying; Health conditions; Destination countries; Estonians

\begin{abstract}
Abbreviations
FF: Fear of Flying; AH: Airplane Headache; DVT: Deep Vein Thrombosis.

\section{Introduction}

Air travel is an important and often necessary part of life, and the number of passengers has grown significantly. Over one billion passengers travel by plane annually, and destination for 50 million of these travelers are developing countries $[1,2]$. Air travel is usually the fastest and sometimes the only way to reach a desired destination. Previously, it has been shown that during flights people might experience headache [3] and earache [4] due to changing pressure. On longer flights, there is a risk of thrombosis [5]. The Fear of Flying (FF) is quite frequent [6], and the chance of transmission of infectious diseases is possible $[1,7]$. Health risks in a foreign country depend on the destination, but travelers are more likely to suffer from sunburns [8], diarrhea [9-11], dehydration [11], and other possible infections $[1,12,13]$.
\end{abstract}

As the number of Estonian travelers is also rising, this kind of knowledge for improving guidelines for preventing health risks are important. Evidence-based information about health problems associated with plane trips in aircraft and in destination countries are deficient. The information about favorite destinations and the advice that travelers get from travel agencies are lacking. Therefore, we aimed to determine the health problems associated with air travel and stays in a foreign country. Also, we tried to determine the flying frequency, favorite destinations, measures to prevent or treat health problems, and sources of information.

\section{Methods}

The study was carried out at Tartu Health Care College (Estonia) and was conducted in the www.connect.ee environment as a webbased questionnaire survey between October 2015 and February 2016. In the survey, adult Estonian citizens were included if they had experienced at least one airplane trip in the last five years during which they went on a vacation and/or to enjoy culture. The occurrence of air travel-related health problems was observed separately on flights of $<1$ (short), 1-4 (medium) and $>5$ (long) hours. The sample size was planned to be 1,000 participants, which would represent approximately $0.1 \%$ of the Estonian population. Approval of the Research Ethics Committee of the University of Tartu was obtained (250/T-5, 8 October 2015). 
Page 2 of 7

The software program Sigma Plot for Windows $11.0(\mathrm{GmbH}$ Formation in Germany) was used for statistical analysis. The results are presented as the means with standard deviations $( \pm \mathrm{SD})$, proportions with percentages, or as odds ratios (OR) with 95\% Confidence Intervals (CI). Continuous data were compared with a t-test or MannWhitney test and proportions with the $\chi^{2}$ test, as appropriate. The $p$ value of $<0.05$ was considered significant.

\section{Results}

\section{Study population and travel frequency}

A total of 1,014 people were included (women=83.4\%). The mean age of respondents was $29.2 \pm 10.8$ years, and most had secondary $(35.6 \%)$ or higher $(44.6 \%)$ education. Those who had experience with air travel usually flew by plane more than once a year. In terms of plane trips per year, almost half of the participants (51.4\%) travelled by air at least 1-3 times; one third (31.9\%) less than once; one tenth (9.1\%) 4-6 times, and $7.7 \%$ more than seven times. People who traveled less than once a year were younger compared with those whose flying experience was more than seven times a year (average age $25.8 \pm 8.8$ vs. $31.7 \pm 11.4$, respectively; $\mathrm{p}<0.001$ ).

\section{Health problems during air travel}

The main problems during flight are earache and headache, but also five cases of FF emerged. Health problems occurred more rarely on shorter and long-haul flights compared to medium flights (Figure 1). Earache occurred in $21 \%, 33 \%$ and $18.2 \%$ of passengers during flights of $<1,1-4$ and $>5$-hours duration, respectively, and the chance to get this painful disorder was almost twice as frequent on flights of 1-4 hours compared with flights of $<1$ and $>5$ hours. The second health problem during the 1-4-hour flights was headache. Five cases $(0.5 \%)$ of flight thrombosis occurred, all on long-distance flights. Passengers without health problems made up about one fifth of the total in each of the three flight length groups: namely, $22.6 \%$ in flights of $<1$ hour, $21.7 \%$ in flights $1-4$ hours and $18 \%$ in flights $>5$ hours.

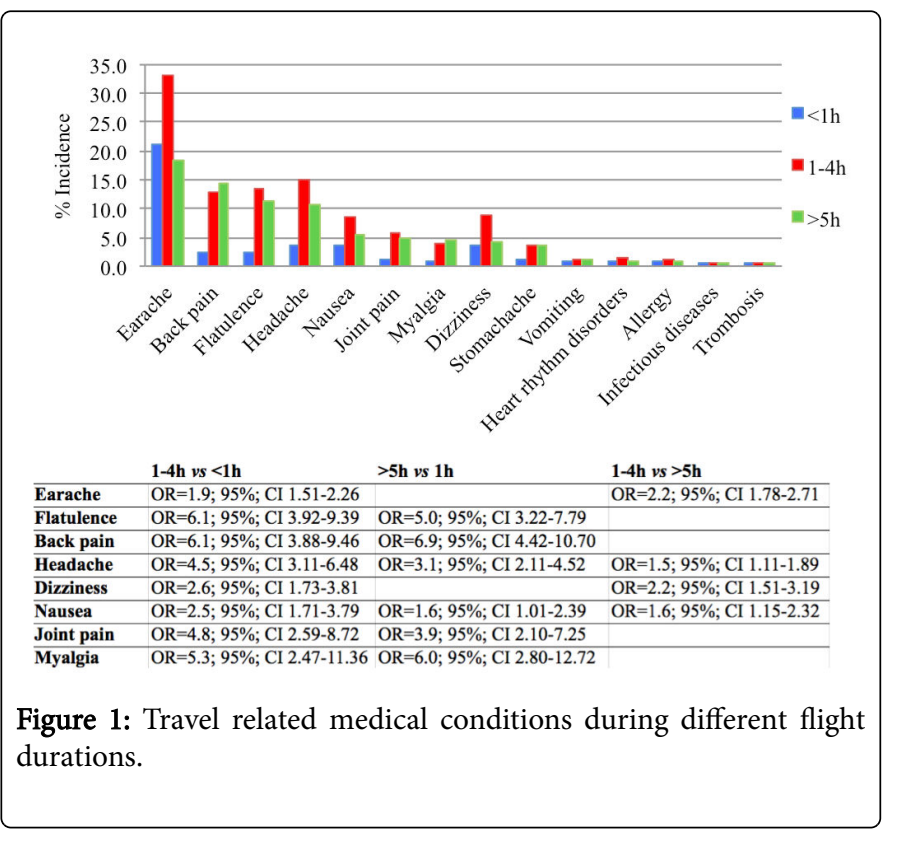

FF was attested to by a third of our participants (32.3\%); $33 \%$ of women and $28.6 \%$ of men. Figure 2 illustrates the manifestations of FF: palpitations $(55.7 \%)$, pre-flight insomnia (53.5\%), and sweating (36.7\%).

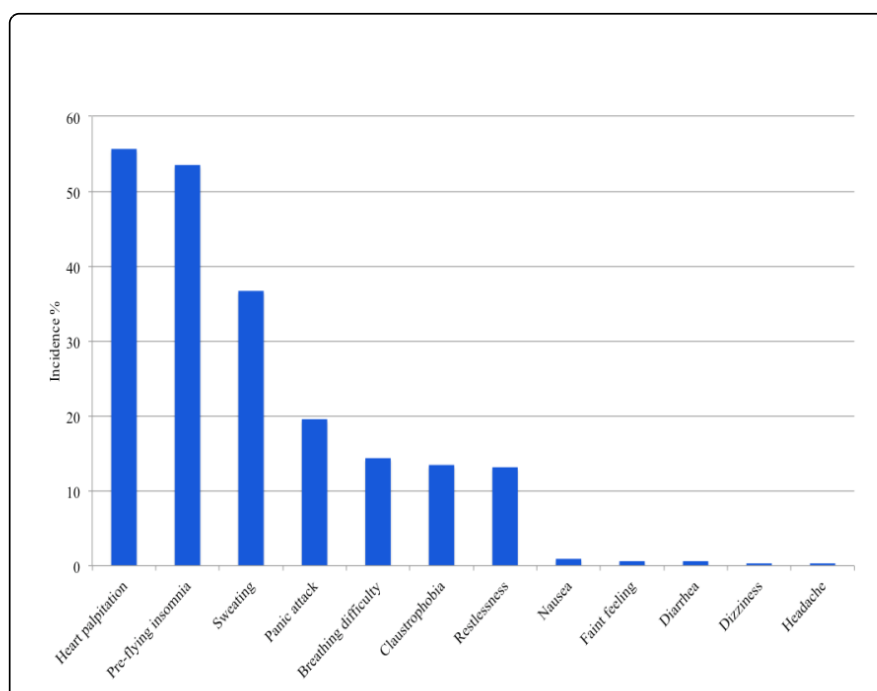

Figure 2: Symptoms of fear of flying.

FF was influenced by the media (57.5\%) but also by fear of heights (36.7\%). The most common ways to avoid FF were listening to music (40.4\%), drinking alcohol (24.2\%), using medications (17.4\%), and, in almost a third of participants (32.1\%), doing nothing. Men consumed alcohol more frequently than women (Figure 3 ).

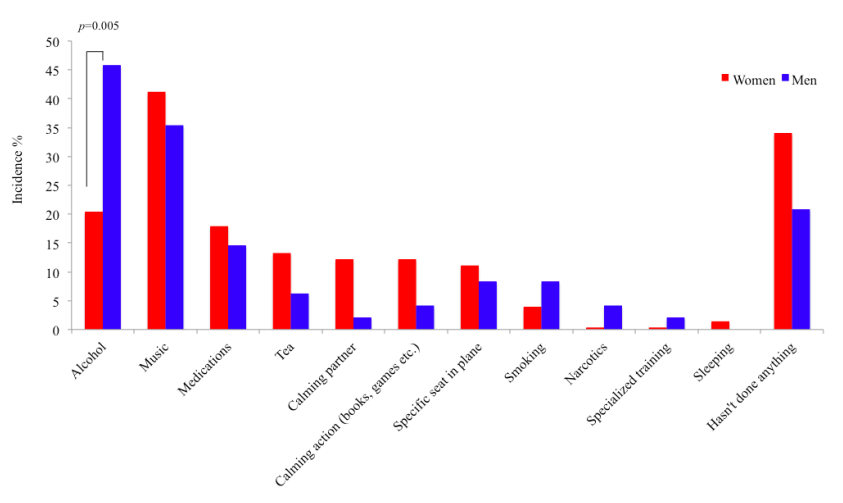

Figure 3: Measures to avoid fear of flying.

\section{Health problems in foreign countries}

Our participants visited a total of 59 different destinations, and the most frequent were Turkey (11\%), Spain (9.6\%), Italy (8.3\%) and Great Britain (7.5\%) (Figure 4). Other countries (please see Figure 4 legend) 
were visited by $19.1 \%$ of study participants, each country by fewer than by 10 persons.

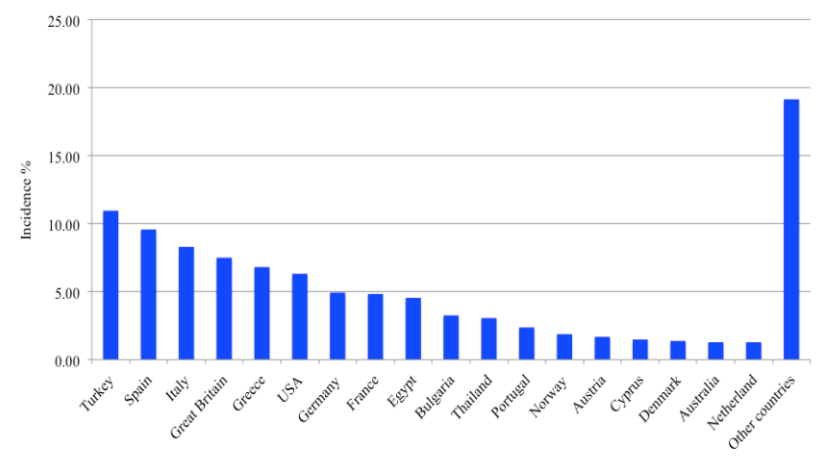

Figure 4: Preferred destinations, Other countries included Afghanistan, Albania, Armenia, Bali Island, Belgium, Bosnia and Herzegovina, Cambodia, China, Czech Republic, Croatia, Curacao, Dominican Republic, East Timor, Finland, Gambia, Georgia, India, Indonesia, Ireland, Jamaica, Hungary, Japan, Macedonia, Malaysia, Malta, Mexico, New Zealand, Poland, Puerto Rico, Russia, Sicily, Singapore, Slovenia, Sri Lanka, Sweden, Switzerland, Tunisia, Ukraine, United Arab Emirates, and Vietnam.

Among these destinations were several exotic countries, which suggest that Estonians try to discover new places. In general, almost a fifth of our respondents (19.2\%) experienced health disorders in their destination countries. Women who experienced health problems were, surprisingly, younger than those who did not have this kind of experience (mean age $27.5 \pm 9.6$ vs $29.6 \pm 11.0 ; \mathrm{p}=0.04$ ). The most common health problems in destination countries were diarrhea (35.4\%), sunburns (20\%) and strong headache (19.5\%) (Figure 5).

Diarrhea (35.4\%) was the most frequent affliction of Estonian tourists in Bulgaria, Turkey and Egypt and was more frequent among men comparing with women $(40 \%$ vs $16.4 \% ; \mathrm{p}=0.022)$. Sunburns appeared mostly among travelers visiting Thailand, and headache (19.5\%) occurred mostly in people who visited Spain. Influenza-like symptoms occurred in $38 \%$ of women who reported any health problems. Frequently, the symptoms related to common cold occurred in Great Britain (60\%) (Figure 5).

\section{Preventative and treatment measures}

Only half (52.9\%) of travelers recognized the health risks associated with destination counties prior to their trip, while $30.7 \%$ were not aware of them. Advice and information about the health-risks in destination countries were mostly obtained from the Internet $(46.5 \%)$, media $(24.7 \%)$, and friends $(20.4 \%)$; in only $1.6 \%$ of instances did it come from a travel agency.

Most problems that accompany travelling are country-specific. Our participants believed the possible causes of their health problems were local food $(34.4 \%)$, change of environment $(25.6 \%)$, lack of drinking water $(20.5 \%)$, change of menu (different from their regular menu,
$18.5 \%)$ and not using sunscreen (17.4\%). To resolve these problems, about half of travelers used relaxing (41.5\%) and medication (59.5\%). One fifth of our travelers got sunburn, and the main reason for this was not using sun creams.

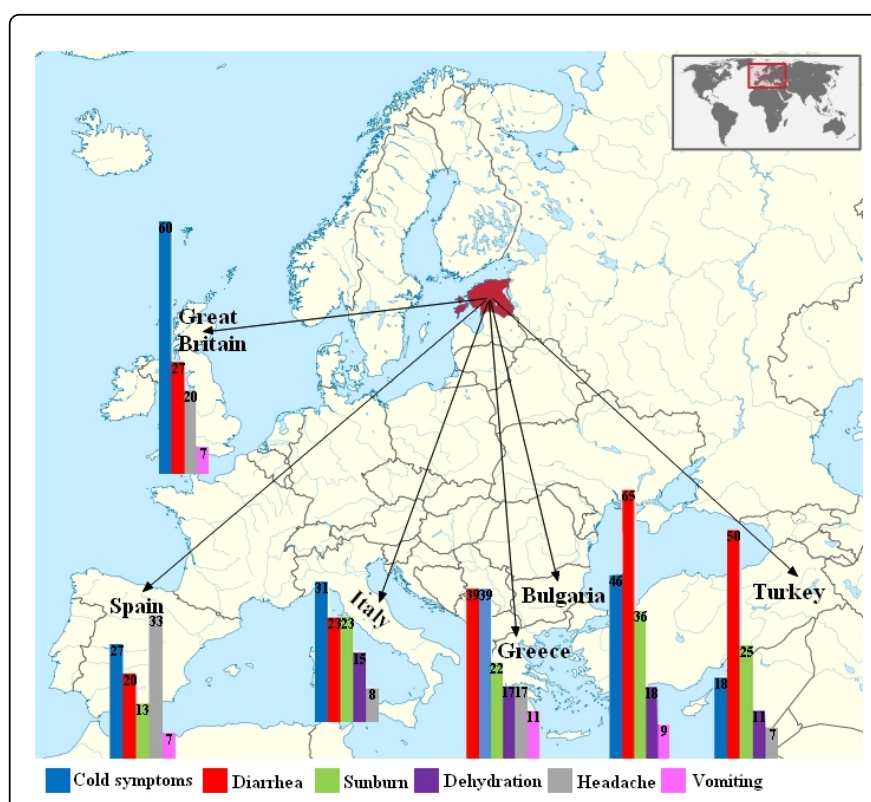

Figure 5: The mean health problems that occurred in the destination countries of the European region. The numbers show the proportion of persons (percentage) with health problems in a particular country.

Prior to their trips, $74.5 \%$ of the respondents obtained health insurance, most frequently prior to visits to Bulgaria, Turkey, Austria and Cyprus. Only $1.9 \%$ of the respondents had been vaccinated; frequently acquired vaccinations were against Hepatitis-A (9.6\%) and B (8\%) and typhoid fever (4.8\%). Among travelers, $10.5 \%$ had been vaccinated in the past but not prior their current trip, and $87.7 \%$ believed that the target country did not require it. Based on vaccination recommendations of the Estonian Health Board, it emerged that over a fifth of respondents $(21.4 \%)$ incorrectly thought that vaccination is not required, which means that the vaccination level was lower than recommended. For example, in Estonia, prior to visiting Bulgaria, Turkey, Egypt, Italy, Australia, and Holland, vaccination against Hepatitis $\mathrm{A}$ is recommended, but only $13.8 \%$ of persons visiting these countries had complied. The destinations most frequently visited by travelers without the recommended vaccinations were Bulgaria (3\%) and Turkey (12.6\%).

\section{Discussion}

The results of this study are based mostly on the experiences of young Estonian women with secondary and higher education. Frequent travel experiences among women have been shown previously [14], and women aged 18-29 years are also known to be frequent visitors of the social network Facebook, where the information of the survey was uploaded. We can also speculate that people with higher education have more income, allowing them to travel more frequently. For example, in Estonia, according to formal statistical data (2014), a person with a basic education earns about two 
times less than a person with a higher education. So, travelling by plane is more affordable for more educated people.

Air travel is often a way of life, but it might influence health and well-being. While previous studies have usually concentrated on one specific disorder like flight thrombosis [15,16], headache [17] and earache $[4,18]$, we tried to achieve an overview of almost all the frequent disorders that can occur during a flight. Our work is consistent with previous studies, and we do not present any new concepts here $[3,4,6,19]$. Our study confirmed that the most common health disorder during flight is earache [4], but frequent headache and five cases of FF emerged.

However, the results of our study showed that most health problems occurred on medium duration flights. It is not easy to explain this, and it might be associated with our study design (questionnaire). We asked respondents to fill in the table noting the duration of any flight during which a health problem emerged, but we cannot present the flight length-stratified data. If most people have the experience of a 1-4-hour plane trip, it is obvious that problems would occur more often in this group; statistics confirm our results. On the other hand, the duration of the flight might relate to health problems. For example, the most frequent painful disorder on those flights was earache, and that is experienced by passengers who cannot regulate their middle ear pressure in response to changing cabin pressure during take-off or, more frequently, during landing of the aircraft [4]. We can only speculate that during short flights in a small plane, the ascending and descending periods are also quite short and that planes used for longhaul flights are sufficiently high-grade so that the range of air pressures onboard the aircraft is not experienced.

The second health problem was headache. The syndrome called "Airplane Headache" (AH) is quite new and was first described in 2004. A real AH lasts generally at least 20 minutes and is qualified by at least two characteristics: it is either one-sided, intense, stabbing, throbbing pain or consistent pain that is localized in the per orbital region [3]. The AH has also been described as a disorder that appears exclusively in relation to airplane flights, in particular during the landing phase and, based on the findings of Mainardi et al. [17], 86.6\% of the 75 passengers with suspected AH truly had this. We did not ask about and analyze headache in association with take-off and landing in order to diagnose a real $\mathrm{AH}$, but there are many reasons for probable cause of this kind of headache: stress, tiredness, lack of humidity and oxygen in the aircraft cabin, noise and vibration, subfreezing temperatures, etc. Therefore, it is very likely that this headache occurs specifically during long and medium flights. However, previous studies have shown that low humidity in the aircraft cabin is not noticeable on flights shorter than 3-4 hours [20], but the reason needs future clarification [21].

Some air travelers are at risk of developing Deep Vein Thrombosis (DVT) caused by prolonged sitting. This condition, when a small blood clot develops in the deep veins of usually the leg or pelvis, appears during or soon after travelling [22]. As previously reported, the frequency of flight thrombosis is approximately 1-2 cases per 1,000 passengers per year [22]; we can confirm this, as there were five cases and our study reflects the experience of approximately 1,000 passengers during last five years. All cases occurred on long-distance flights, and flight duration of longer than 6 hours is a known risk factor [19]. As has been shown previously, DVT is not only a problem associated with the tight confines of an aircraft cabin, as it can occur in other modes of transportation or with prolonged sitting [7].
As the condition may manifest some weeks or a month after the trip, it is thought that flight thrombosis is underdiagnosed [5]. DVT is not directly life-threatening, but the complication of a pulmonary embolism can be [22]. A study carried out in New Zealand [23] found that the chance of a pulmonary embolism on flights longer than eight hours was eight times higher than on flights shorter than three hours (95\% CI 1.1-55.1). As the effect of behavioral factors on the risk of DVT is limited [15], other factors that predispose individuals to DVT must be considered, such as surgery within 6 weeks, oral contraception, pregnancy, cigarette smoking, etc. [24]; these are important.

FF can cause distress to passengers, and this was attested to by a third of our participants. It is a common condition (suffered by $2.5 \%-$ $40 \%$ of the population) that is characterized by pronounced, lasting and strong FF [25]. This heterogeneous phenomenon is influenced by complex psychological, social and physiological factors [25]. In our participants FF caused palpitations, pre-flight insomnia, and sweating, as expected [26]. Results of a study by Kahan et al. [27] show that the most frequent concerns were fear of a crash (48.4\%) and agoraphobia (26\%). Factually, flying by planes is believed to be safer than staying at home, driving or taking the train [25].

FF among of our participants was prevailingly initiated by the media. However, the results of previous studies that looked at the influence of the media as a contributing factor to informational acquisition of FF are controversial: some studies show negligible effects, others show a measurable impact [25]. Ekeberg et al. [28] show that after the September 11 attacks in the USA, FF among women rose up by $8.7 \%$. As the most common ways to avoid FF among our participants were listening to music, using medications and drinking alcohol, it has been suggested that the golden rule for FF patients is the avoidance of alcohol [6]. The most effective interventions in the treatment of $\mathrm{FF}$ are those that are based on an accurate functional assessment on an individual level [29].

Over the past six decades, tourism has become one of the largest and fastest-growing economic sectors in the world [30]. The growing mobility of people and the popularity of plane transportation have amplified the potential for health problems, not only during travel but also issues that are associated with the destination country [1]. Our participants visited a total of 59 different destinations that mostly were not neighboring countries but also were not very distant from Estonia. Among them were several exotic countries, which suggest that Estonians try to discover new places. Recently, it has been shown that modern tourism is closely linked to development and encompasses a growing number of destinations in countries like China, South Africa, South Korea and Indonesia [30]. However, these countries are not among the top 18 choices of Estonians.

Therefore, travel must be interesting, exiting and good for mental and physical wellbeing, but it can also be harmful to a traveler's health. The health problems that may appear in different countries are dependent on climate, level of hygiene, the behavior of people, etc. In general, almost one fifth experienced health disorders in destination countries. Research that has examined mortality indicated that about one fourth of deaths during travel are caused by injuries and accidents; and research on morbidity has suggested that diarrhea is the most common problem to be encountered [13]. As Estonian travelers, like other North-Europeans, prefer southern countries, the health problems in destination countries were analogous to results published previously $[8,31,32]$. The most common issues were diarrhea, sunburns and strong headache. 
As has previously been shown, up to $50 \%$ of travelers have experienced a gastrointestinal disorder [2], and among those from developed countries who visit developing countries, more than $60 \%$ have reported this issue [33]. In comparison, we can say that our results are quite good. This can be explained by destinations that our travelers visited more frequently. According to Steffen [33], only Turkey and Egypt belong in the high-risk zone. If there is an additional and large risk of infection in Africa, Central and South America and in Asia, these areas (except Thailand) were rarely visited. Travelers' diarrhea is usually caused by different bacteria, viruses or parasites [2]. Most likely, men are less conscious of what they eat and do not follow the rules of hygiene. Although the spontaneous cure of traveler's diarrhea occurs commonly after 3-4 days, and it usually is not a severe illness, the symptoms accompanying the disease (diarrhea, fever, nausea, vomiting, and cramps) [33] are uncomfortable and might disturb the trip.

Sunbathing, swimming and other outdoor pursuits remain popular activities among travelers, but too much exposure to sunlight can also cause health problems. People travel more frequently to obtain a suntan, a change that is caused by the sun's UVB-rays, but excessive sunbathing may cause erythema, skin burns and cancer [34]. However, the sun also has a positive effect through the synthesis of vitamin D and promotes higher self-esteem due to a better complexion. We cannot contradict the emotional effect of sun, sand and sea. As expected, sunburns appeared mostly among participants visiting southern countries such as Thailand. As the sunlight is very intense there, the risk of skin burns in light-skinned tourists like Estonians is a logical outcome.

The experience of headache occurred mostly in visitors to Spain, and common cold symptoms were noted in visitors of Great Britain. It is known that the English climate promotes infections in the respiratory tract [35]; unfortunately, we did not specify if tourists travelled during winter or summer. Based on Centers for Disease Control and Prevention (CDC), respiratory infections occur in up to $20 \%$ of all travelers and are almost as common as travelers' diarrhea [36]. Viruses causing respiratory system infections are spread by the way of droplets through coughing and sneezing, and outbreaks among passengers in hotels and tourist groups in locations such as airports and hotels can occur [37]. The risk of real influenza depends on the season and destination. As the season for influenza differs for the northern and southern hemispheres, people from northern countries who travel to tropical or subtropical regions have a year-round risk of influenza [36].

To prevent the health problems that occur in the travelers who go abroad, risk assessment has to be undertaken as of part of a pre-travel consultation [13]. The lack of information among travelers emerged: only half recognized the health risks associated with their destination counties prior to their trip. In Estonia, the travel agency is obligated to inform the traveler of the following: 1) the danger of infectious diseases in the target country, 2) the efficacy and availability of immunizations or drugs, 3) the possibility of getting medical advice before a trip, receiving medical attention during the trip and needing a health check after the trip. Results of our survey revealed that travel agencies were a source of information for only a few travelers.

For Estonian people, information sources regarding travel are mostly the Internet, the media and friends. These results differ from Australian, where the role of the Internet was only $1.5 \%$ (10/670), and the most frequent source to get travel advice was a general practitioner (39\%) [38]. To give adequate advice on travel health requires good knowledge about local health hazards overseas and about the effectiveness of immunizations and any other kind of prophylaxis [39]. As the Estonian travel agencies probably are not working at the level expected, the use of Internet is recommended. The detailed official information about infection risks in all destination countries can be found in regularly updated sources, which, in Estonia, is the National Health Board; information can also be obtained from the World Health Organization webpage.

Most of the problems that accompany travelling were countryspecific. About half of travelers resolved the problems with relaxation and medication. We did not request that they specify for what health problems specific medications were used. Therefore, we cannot decide upon the efficiency of these measures. As the most frequent problem was diarrhea, the avoidance of possible contaminated foods and beverages is necessary. As the problem had already emerged, we can suppose that some kind antibiotics were used. As diarrhea associated with international travel is commonly caused by enterotoxigenic and enteroaggregative $E$. coli, it has previously have been shown that chemoprophylaxis may reduce the risk and that empirical antibacterial therapy is effective in shortening illness [40]. On the other hand, as the causative factors of some kind of viruses (e.g., norovirus) are arising for which therapy by antibiotics is not needed and as the problem of antibiotic resistant-strains is largely known, this recommendation is impugnable in all cases, especially in short-term healthy travelers [31].

In southern countries, sunburns were frequent for the reason of not using sun creams. Currently, there are many components that can significantly affect the protective ability of a sunscreen 's. The ones that we need to know include sun protection factor number, amount of initial sunscreen applied, season, time of day, water exposure, skin type, etc. [41]. Additionally, especially for travelers to regions with a high UV index, recommendations included wearing sunglasses and protective clothing and practicing responsible sun exposure behaviors [41]. The avoidance of sun exposure during the midday could reduce accumulated ultraviolet radiation (UVR) doses markedly [32]. As the high exposure of solar UVR is the main cause of the skin cancer [34], Petersen et al. [32] believe that minimizing the number of "sunbathing" holidays would reduce of skin cancer incidence in Europe.

Three fourths of the respondents obtained health insurance prior to their trip, although this travel document is intended to cover medical expenses that are incurred while travelling. There is no need to vaccinate prior visiting any country, but the vaccination level was lower than recommended. We did not ask the reason for not vaccinating, but according to Henderson et al. [38], it might be explained by lack of knowledge, the high price of a vaccine or refusal. Our travelers were frequently vaccinated against Hepatitis- $\mathrm{A}$ and $-\mathrm{B}$ and typhoid fever. These infections belong to the top 7 of the world's 10 worst travel diseases [13], and vaccination against these diseases are most frequent among Australian travelers [38]. Pre-travel vaccination against Hepatitis-B among our travelers was quite low, which might be associated with earlier vaccination in childhood (this measure has been part of the Estonian vaccination plan since 2003). The most effective prevention method for influenza is also vaccination $[37,42]$, but we have no data that anyone of our study group had gotten such a vaccination. Vaccines are important public health measure but are not always useful for the last-minute traveler.

People without immunizations might contract an infectious disease in a target country. For example, in 2015, there were three cases of Hepatitis-A that were brought to Estonia from foreign countries. One 
aspect of travel health is handling travelers after their return, but presumably only a few travelers with health-problems that occurred during a trip turn to doctors to certify the diagnose. Therefore, the real number of diseases brought in is not known. Most likely, some of the people with infectious diseases are not truly treated and may be a source of infection.

There was some study limitations described previously. First, the questionnaire was available as a web-based instrument only; this study presented findings for only those who used the Web and for whom the questionnaires were accessible. For most of Estonians, web-based access is common although, perhaps, not among some older people. Second, the premise of taking part of this study was the experience of at least one air-trip during the last five years for the purpose of vacation and/or to enjoy culture. If there were more than one trip to choose from (and there was), travelers had to select one of them to use for describing health-problems associated with the destination country. Most likely, the participants choose the country that, for them, was in some way special. That remarkableness might be associated with health-problems for them, and those might be overstated. On the other hand, approximately $0.1 \%$ of Estonians took part in this study; this could give an overview of the situation.

\section{Conclusions}

Young Estonians, especially women, preferred destinations with a warmer climate that were not so distant (Turkey, Spain, Italy); usually flew by plane more than once a year. Health problems during flight (ear-, back-, and headache and those associated with FF) were quite frequent and occurred usually during flights of medium length (1-4 hours). The most common ways to avoid FF were listening to music and, especially for men, consuming alcohol. The frequent health disorders in destination countries were characteristic for northern tourists visiting southern countries (diarrhea, sunburns and strong headache); these were resolved by relaxing and taking medication. The behavior of the Estonian people is influenced mostly by social media (the Internet); information from travel agencies is lacking.

\section{Acknowledgments}

The publishing is supported by European Union Regional Development Fund.

\section{References}

1. Mangili A, Gendreau MA (2005) Transmission of infectious diseases during commercial air travel. Lancet 365: 989-996.

2. Patel RR, Liang SY, Koolwal P, Kuhlmann FM (2015) Travel advice for the immunocompromised traveler: prophylaxis, vaccination, and other preventive measures. Ther Clin Risk Manag 11: 217-228.

3. Mainardi F, Lisotto C, Palestini C, Sarchielli P, Maggioni F, et al. (2007) Headache attributed to airplane travel ("airplane headache"): first Italian case. J Headache Pain 8: 196-199.

4. Kanick SC, Doyle WJ (2005) Barotrauma during air travel: predictions of a mathematical model. J Appl Physiol 98: 1592-1602.

5. Stricker H (2006) Clotting in the air. Lancet 367: 792-794.

6. Van Gerwen LJ, Diekstra RF, Arondeus JM, Wolfger R (2004) Fear of flying treatment programs for passengers: an international update. Travel Med Infect Dis 2: 27-35.

7. DeHart RL (2003) Health Issues of a Air Travel. Annu Rev Public Health 24: 133-151.
8. Boldeman C, Branstrom R, Dal H, Kristjansson S, Rodvall Y, et al. (2001) Tanning habits and sunburn in a Swedish population age 13-50 years. Eur J Cancer 37: 2441-2448.

9. Choe YJ, Choe SA, Cho SI (2017) Importation of travel-related infectious diseases is increasing in South Korea: An analysis of salmonellosis, shigellosis, malaria, and dengue surveillance data. Travel Med Infect Dis 19: 22-27.

10. Gautret P, Schlagenhauf P, Gaudart J, Castelli F, Brouqui P, et al. (2009) GeoSentinel Surveillance N. Multicenter EuroTravNet/GeoSentinel study of travel-related infectious diseases in Europe. Emerging Infect Dis 15: 1783-1790.

11. De Truchis P, De Truchis A (2007) Acute infectious diarrhea. Presse Medicale 36: 695-705.

12. Dahlgren AL, DeRoo L, Steffen R (2006) Prevention of travel-related infectious diseases: knowledge, practices and attitudes of Swedish travellers. Scand J Infect Dis 38: 1074-1080.

13. Sushma R, Nagabhushana D (2012) Travellers Health Through "Emporiatrics". J Clin Diagn Res 6: 1324-1327.

14. Rind E, Shortt N, Mitchell R, Richardson EA, Pearce J (2015) Are income-related differences in active travel associated with physical environmental characteristics? A multi-level ecological approach. Int J Behav Nutr Phys Act 12: 73.

15. Schreijer AJ, Cannegieter SC, Doggen CJ, Rosendaal FR (2008) The effect of flight-related behaviour on the risk of venous thrombosis after air travel. Br J Haematol 144: 425-429.

16. Toff WD, Jones CI, Ford I, Pearse RJ, Watson HG, et al. (2006) Effect of hypobaric hypoxia, simulating conditions during long-haul air travel, on coagulation, fibrinolysis, platelet function, and endothelial activation. JAMA 295: 2251-2261.

17. Mainardi F, Lisotto C, Maggioni F, Zanchin G (2012) Headache attributed to airplane travel ('airplane headache'): clinical profile based on a large case series. Cephalalgia 32: 592-599.

18. Mirza S, Richardson H (2005) Otic barotrauma from air travel. J Laryngol Otol 119: 366-370.

19. Philbrick JT, Shumate R, Siadaty MS, Becker DM (2007) Air travel and venous thromboembolism: a systematic review. J Gen Intern Med 22: 107-114.

20. Nagda NL, Hodgson M (2001) Low relative humidity and aircraft cabin air quality. Indoor Air 11: 200-214.

21. DeHart RL (2003) Health issues of air travel. Annu Rev Public Health 24: 133-151.

22. Bagshaw M (2001) Traveller's thrombosis: a review of deep vein thrombosis associated with travel. The Air Transport Medicine Committee, Aerospace Medical Association. Aviat Space Environ Med 72: 848-851.

23. Parkin L, Bell ML, Herbison GP, Paul C, Skegg DC (2006) Air travel and fatal pulmonary embolism. Thromb Haemost 95: 807-814.

24. Reid R, Leyland N, Wolfman W, Allaire C, Awadalla A, et al. (2011) SOGC clinical practice guidelines: Oral contraceptives and the risk of venous thromboembolism: an update: no. 252, December 2010. Int J Gynaecol Obstet 112: 252-256.

25. Oakes M, Bor R (2010) The psychology of fear of flying (part I): a critical evaluation of current perspectives on the nature, prevalence and etiology of fear of flying. Travel Med Infect Dis 8: 327-338.

26. Bornas X, Llabres J, Tortella-Feliu M, Fullana MA, Montoya P, et al. (2007) Vagally mediated heart rate variability and heart rate entropy as predictors of treatment outcome in flight phobia. Biol Psychol 76: 188-195.

27. Kahan M, Tanzer J, Darvin D, Borer F (2000) Virtual Reality-Assisted Cognitive-Behavioral Treatment for Fear of Flying: Acute Treatment and Follow-Up. CyberPsychol Behav 3: 387-392.

28. Ekeberg O, Fauske B, Berg-Hansen B (2014) Norwegian airline passengers are not more afraid of flying after the terror act of September 11. The flight anxiety, however, is significantly attributed to acts of terrorism. Scand J Psychol 55: 464-468. 
Citation: Parm U, Must G, Kindsiko K, Tamm AL (2018) Health Conditions Related to Air Travel and Destination Country. J Health Educ Res Dev

Page 7 of 7

29. Oakes M, Bor R (2010) The psychology of fear of flying (part II): a critical evaluation of current perspectives on approaches to treatment. Travel Med Infect Dis 8: 339-363.

30. Pantelescu AM (2012) Trends in International Tourism. Cactus Tourism J 3: 31-35.

31. Belderok SM, van den Hoek A, Kint JA, Schim van der Loeff MF, Sonder GJ (2011) Incidence, risk factors and treatment of diarrhoea among Dutch travellers: reasons not to routinely prescribe antibiotics. BMC Infect Dis 11: 295.

32. Petersen B, Triguero-Mas M, Maier B, Thieden E, Philipsen PA, et al. (2015) Sun behaviour and personal UVR exposure among Europeans on short term holidays. J Photochem Photobiol B 151: 264-269.

33. Steffen R (2005) Epidemiology of traveler's diarrhea. Clin Infect Dis 41: S536-540.

34. Chang YM, Barrett JH, Bishop DT, Armstrong BK, Bataille V, et al. (2009) Sun exposure and melanoma risk at different latitudes: a pooled analysis of 5700 cases and 7216 controls. Int J Epidemiol 38: 814-830.

35. Mourtzoukou EG, Falagas ME (2007) Exposure to cold and respiratory tract infections. Int J Tuberc Lung Dis 11: 938-943.
36. LaRocque RC, Ryan ET (2017) Respiratory Infections. Travelers' Health. Chapter 2. The Pretravel Consultation. Self-Treatable Conditions.

37. Goeijenbier M, Van Genderen P, Ward BJ, Wilder-Smith A, Steffen R, et al. (2017) Travellers and influenza: risks and prevention. J Travel Med, p: 24.

38. Henderson J, Harrison C, Bayram C, Britt H (2015) Travel advice and vaccination. Australian Family Physician 44: 14-15.

39. Sushma R, Nagabhushana D (2012) Emporiatrics: the travellers medicine. Iran J Public Health 41: 133.

40. DuPont HL (2007) Therapy for and prevention of traveler's diarrhea. Clinical Infectious Diseases: An Official Publication of the Infectious Diseases Society of America 45: S78-84.

41. Diaz JH, Nesbitt LT (2013) Sun exposure behavior and protection: recommendations for travelers. J Travel Med 20: 108-118.

42. Pye J (2010) Reducing the risk of respiratory disease in overseas travellers. Nurs Stand 24: 50-56. 